\title{
A proteomic approach for exploring biofilm in Streptococcus mutans
}

\author{
Asad Ullah Khan*, Barira Islam, Shahper N Khan, Mohammad Akram \\ Interdisciplinary Biotechnology Unit, Aligarh Muslim University, Aligarh-202002, India; Asad Ullah Khan - Email: asad.k@rediffmail.com,
} asadukhan72@gmail.com; Phone: 00919837021912; Fax: 0091-571-2721776; *Corresponding author

Received February 07, 2011; Accepted February 11, 2011; Published February 15, 2011

\begin{abstract}
:
Biofilm formation by Streptococcus mutans is considered as its principal virulence factor, causing dental caries. Mutants of S. mutans defective in biofilm formation were generated and analyzed to study the collective role of proteins in its formation. Mutants were characterized on the basis of adherence to saliva-coated surface, and biofilm formation. The confocal laser microscopy and scanning electron microscopy images showed that the control biofilms had cluster of cells covered by layer of exo-polysaccharide while the biofilms of mutants were thin and spaced. Two-dimensional protein electrophoresis data analysis identified 57 proteins that are either up (44 proteins) or down (13 proteins) regulated. These data points to the importance of up and down regulated proteins in the formation of biofilm in Streptococcus mutans.
\end{abstract}

\section{Background:}

Streptococcus mutans is now regarded as the principal microbiological aetiological agent of dental caries and a target of novel preventive strategies. Beyond initial adherence, it appears that a variety of genes are required for the proper maturation of biofilms formed by $S$. mutans and other oral streptococci. By the use of specific- and randommutagenesis strategies, many different types of genes that are required for these organisms to transition from adherent microcolonies to complex, three-dimensional biofilms have been identified. These include those for intercellular communication systems and environmental sensing systems, components of the general stress response pathway involved in protein repair and turnover, global regulators of carbohydrate metabolism, and adhesion-promoting genes [1].

Interestingly, many of the genes identified to affect biofilm formation affect the expression of a large panel of genes, many of which are either unidentified or have no known function [2]. There are a large number of hypothetical proteins of as-yet-unknown function present in the annotated genome of $S$. mutans (http://www.stdgen. lanl.gov/oragen). Among these proteins, there could be other unknown surface proteins that play an important role in adhesion and maturation of biofilms. Techniques like 2D protein analysis and microarray are now being used to screen out the expression of all genes involved in the formation of a biofilm [3, 4]. An integrated approach to study protein-function relationship was also employed to study the variation in S. mutans genome [5].

Chemical mutagenesis induces change in morphological and characteristic traits of $S$. mutans, had been demonstrated earlier by Murchison et al. [6, 7]. However, a deeper insight into the effect of EMS on the adherence and biofilm formation by $S$. mutans was lacking. The biofilm formation by $S$. mutans on saliva-coated surface is believed to be a multi-step process [8]. The initial adherence of $S$. mutans on the saliva-coated surface is mainly due to the interaction of its cell-surface adhesin with the salivary agglutinin [9]. The later stages of biofilm formation are marked by active formation of exopolysaccharides that mediate the clumping and effective biofilm formation of S. mutans [10].

The transition from planktonic phase to biofilm phase of $S$. mutans is a resultant of many differential and altered genes [2]. To explore this, we isolated the EMS-induced adherence defective mutants of S. mutans and characterized them on their defects in biofilm formation. The protein profile of the mutants that show defective initial adherence and biofilm formation was then evaluated.

\section{Methodology:}

Bacterial strains:

The strain of Streptococcus mutans UA159 (MTCC\#497), was purchased from Microbial type culture collection (MTCC), Institute of Microbial Technology, Chandigarh

\section{Generation and isolation of mutants:}

Mutants from UA159 strain of $S$. mutans were generated by the method of Murchison et al. [7]. PCR amplification for was performed by the method of Yano et al. [11] to confirm of S. mutans

\section{Glass-dependent adherence and initial adherence of $\boldsymbol{S}$. mutans:}

Glass surface adherence assay was performed by the method of Hamada et al. with slight modifications [12]. For checking the adherence of S. mutans on saliva-coated surface, human saliva from four healthy individuals was collected and clarified by the method of Shellis et al. [13]. Assessment of the adherence of bacteria to saliva coated surface was done on 96 well flatbottomed microtitre plate, using the protocol of Jakubovics et al. with slight modifications [14].

\section{Confocal microscopy:}

The biofilms were developed on saliva-coated glass cover slips for comparison between parent strain and mutants. The 9-well microtitre plate with seeded saliva-coated cover slips, containing $10 \mathrm{ml}$ of TSB with $0.25 \%$ sucrose was inoculated with $100 \mu \mathrm{l}$ of mid-exponential grown cultures of parent strain and mutants BSM3, BSM5 and BSM61 respectively. The 
cover slips were removed and noninvasive confocal analysis was performed as described elsewhere [15].

\section{Scanning electron microscopy:}

The cells were fixed with $25 \%$ SEM grade glutaraldehyde in PBS, pH 7.2 for 15 minutes. Fixed samples were then dehydrated through a graded series of ethanol concentrations, mounted, and sputter coated with goldpalladium. Samples were analyzed by SEM (Hitachi S-3000 N; High Technology Operation, Japan) at several magnifications $(\times 500$ to $\times 13,000)$ at the EM core laboratory of JALMA, Agra.

\section{Isolation of total cellular proteins of Streptococcus mutans:}

Cells of S. mutans UA159 and mutants BSM3, BSM5 and BSM61 were grown at $37^{\circ} \mathrm{C}$ in an anerobic environment up to an OD $600 \mathrm{~nm}$ of 1.5 . The washed cell pellets were resuspended in lysis buffer containing $5 \mathrm{M}$ Urea, $4 \%$ (w/v) CHAPS, $50 \mathrm{mM}$ DTT and $0.5 \%$ (v/v) IPG buffer (pH 4-7) (GE healthcare, NJ, USA), and left on ice for 10 minutes with intermittent sonication. The cell lysate was centrifuged at $12,000 \mathrm{~g}$ for 10 minutes at $4^{\circ} \mathrm{C}$. The supernatant was collected and protein in it was measured by the method of Lowry et al. [16].

\section{Two-dimensional protein electrophoresis:}

The total protein isolated by the above described method was analyzed by 2D electrophoresis using the method of O'Farrell [17]. Gels were then silver stained, digitally scanned and analyzed using automated software Decodon Delta 2D version 3.6. The gel pictures of parent strain and mutants BSM3, BSM5 and BSM61 were loaded on the Delta 2D platform and warped for the 2D image analysis. The spot detection was done using the software and a synthetic fusion image was constructed of all the four images. The spots were transferred from the fusion image to the individual images and were then quantified [18].

\section{Statistical analysis:}

All the values were calculated as the mean of individual experiments in triplicate, compared with control groups. Comparison between the thickness of control and mutant biofilms was done using SPSS 11.0.0 statistical software using one-way ANOVA and posthoc tests (LSD); statistically significant tests were set at a P-value of $<0.05$. Absorbance from adherence assays was evaluated for statistically significant differences using Student's unpaired two-sided $t$ test (assuming unknown and unequal variances).

\section{Discussion:}

The EMS-induced mutagenesis had earlier been done by Murchison et al. $[6,7]$, for the isolation of adherence defective mutants of S. mutans. A similar methodology was followed in this study and mutagenized cells were subcultured four times for isolation of stable mutants, containing chains of genotypically similar cells [6]. In our experiments, the survival frequency after mutagenesis was $14 \%$ whereas Murchinson et al. [6] reported it to be $10 \%$. Nearly $70 \%$ of the picked mutagenized cells showed some amount of decrease in adherence to the glass surface. The S. mutans cells showing nearly or more than $50 \%$ decrease in adherence to the glass surface were selected for further characterization.

The salivary proteins act as a receptor of $S$. mutans as they carry the anchor sites for the wall proteins of $S$. mutans [19]. The initial adherence of selected $S$. mutans mutant cells on the saliva-coated hydrophobic surface, specifically polystyrene microtitre plates was examined for further characterization of the mutants (Table 2 see Supplementary material). $40 \%$ of the isolated mutants showed more than $50 \%$ decrease in initial adherence to saliva-coated polystyrene surface. The decrease in initial adherence of these mutants implies a possible mutation in bacterial proteins that interact with the saliva.

The interaction of $S$. mutans cells with saliva plays a key role in the biofilm formation on the dental plaque [20]. The biofilm formation by $S$. mutans on the saliva-coated surface was reduced in all the mutants (Table 3 see Supplementary material). Also, the biofilm formation was found to be least in the mutants showing the least initial adherence (BSM3) and maximum in the mutants showing maximum initial adherence (BSM61). This implies that the biofilm formation is dependent on the initial adherence of the mutants.

The biofilm formation by $S$. mutans is a phase dependent process. The time-dependent assay (Table 4 see Supplementary material) reveals that the sucrose-independent adherence defective mutants show less biofilm formation $(61 \%$ of the control) even in the initial phase. The initial phase is marked by initial adherence of the bacteria to the substratum, here salivacoated polystyrene plate (8). However, the sucrose-dependent adherence defective mutants show just $1.5 \%$ inhibition at initial phase while showing a distinct inhibition at the multiplication (40\%) and maturation (42\%) phase of biofilm formation. The later stages of biofilm are marked by the synthesis of exopolysaccharide (glucan). The time-dependent analysis of biofilm formation thus implies that sucrose-independent mutants are altered at the stage of saliva-bacteria interaction. However, sucrosedependent mutants showed an alteration in glucan mediated adherence of the cells for the biofilm formation.

The thickness and the architecture of the biofilm of some sucroseindependent mutants were studied by confocal microscopy. Quantitative estimations show that amongst the mutants BSM3 showed the least biofilm formation while BSM61 showed the maximum biofilm formation (Table 5 see Supplementary material). BSM5 also showed stable and relatively more adherence defective character; hence we selected these three sucroseindependent adherence defective mutants for Confocal Microscopic analysis. The micrographs suggest that the biofilm formed by the parent strain covered a larger surface area and had a definite architecture (Figure 1). However, the biofilms formed by the mutants showed more or less spread cells with no distinct pattern of arrangement. The biofilm formed by BSM61 showed more clumped and aggregated cells than mutants BSM3 and BSM5. The thickness of the biofilm was also found to be more for BSM61 than mutants BSM3 and BSM5, possibly due to more clumping and aggregation of the bacteria. Thus the confocal microscopic revelations go well in agreement with the quantitative estimations of biofilms.

The scanning electron microscopy also showed that the biofilms formed by the parent strain have aggregated and clumped bacteria with a cloud of exopolysaccharide surrounding the cells. The apparent biofilm formed by mutant BSM3 has very few cells individually scattered along the surface. The cells were arranged in short chains with absence of exopolysaccharide matrix. The biofilm formed by the mutant BSM61 was very much patchy. The aggregates of cell clumps were separated by large voids, indicating that original biofilm forming ability as of the parent strain was disrupted to some extent by mutation (Figure 2).

The two-dimensional protein electrophoresis of total protein from parent strain and mutants BSM3, BSM5 and BSM61 was carried out to compare the expression of total proteins. The protein profile of parent strain was called as group1 while the protein profile of mutants BSM3, BSM5 and BSM61 was collectively called as group 2 . The ratio of group 2 to group 1 was used to identify the differentially expressed proteins between the parent strain and the mutants. The proteins were identified on the basis of their MW and pI data obtained from www.stdgen.lanl.gov/ oralgen/bacteria/smut/. (Table 1 see Supplementary material).

shows the identification of proteins that are at least five-fold over- or under-expressed in the parent strain and the mutants. The number of these proteins was 57 whereas the total identified spots were 401 . Of these 57 proteins, 13 are expressed more in parent strain than in the mutants. 10 amongst 13 of these proteins could not be identified. Rex A which modulates transcription in response to changes in cellular NADH/NAD $(+)$ redox state is expressed 5.5 folds more in parent strain while amongst group 2, it is more expressed in BSM 61 and least in BSM 3. Two other under expressed proteins in group 2 are ribonucleotide reductase and a conserved hypothetical protein (Table 1 see Supplementary material)

Table 1 shows that 44 proteins are expressed more in group 2 than in group1. Apart from the 3 unidentified proteins, most of these proteins are associated with basic metabolism that takes place in the cell. This can be 


\section{Bioinformation}

accounted as the planktonic cells have more active metabolism than the biofilm cells. Heat shock protein Dna K (spot id 26) was expressed 6.65 fold more in the mutants than in the parent strain. An up regulation of Dna $\mathrm{K}$ by 5.6 folds after radiation had also been reported earlier [21]. Rec A shows a 9.74 folds up regulation in the mutants while it shows 2 fold up regulation after radiation [21]. It might be due to the exposure of cells to chemical mutagen results in constitutive over expression of heat shock protein and Rec A. These proteins are also over expressed in biofilm phase, salt and oxidation stress [22]. 11 proteins are identified to show quite low expression in the parent strain (mean percent volume nearly 0). Cap A, a membrane associated protein, possibly a capsule biosynthesis protein shows a high expression in mutants but insignificant expression in parent strain.
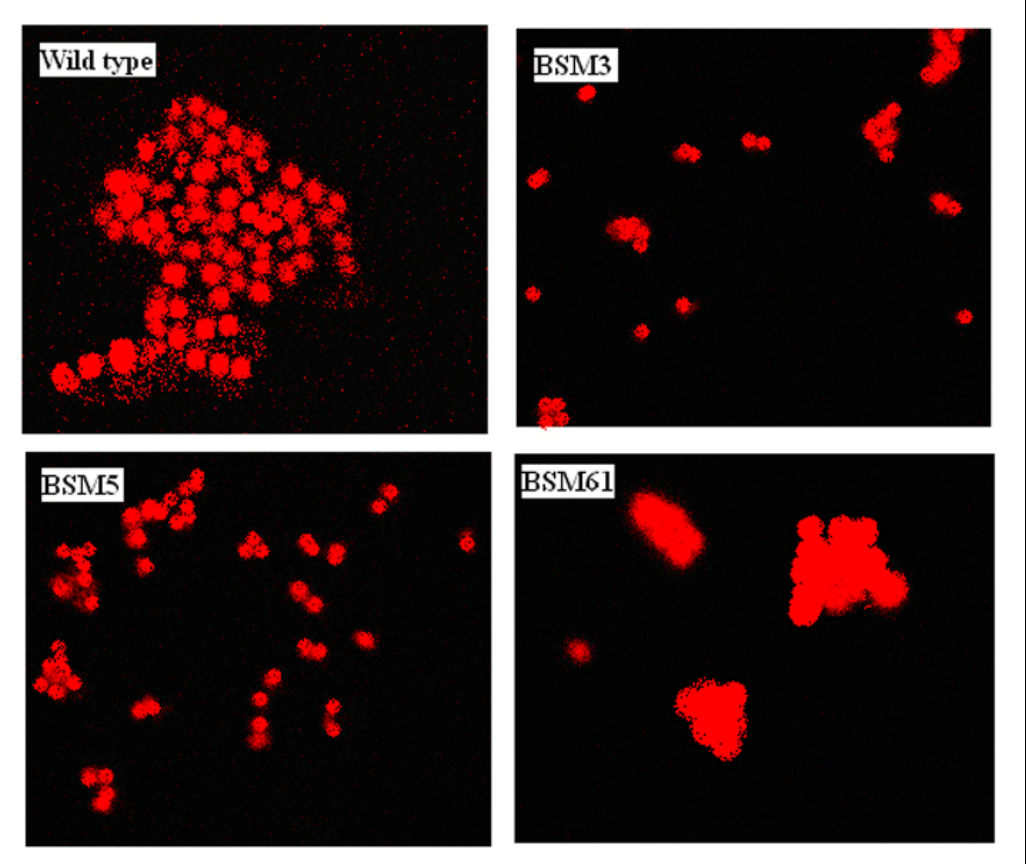

Figure 1: Confocal Laser Scanning microscopy of the parent strain and the sucrose independent mutants; top left panel is control (wild type), top right panel is BSM3 (mutant), bottom left panel is BSM5 (mutant), bottom right panel is BSM61 (mutant). All mutant are showing scattered pattern of cells as compared with wild type.
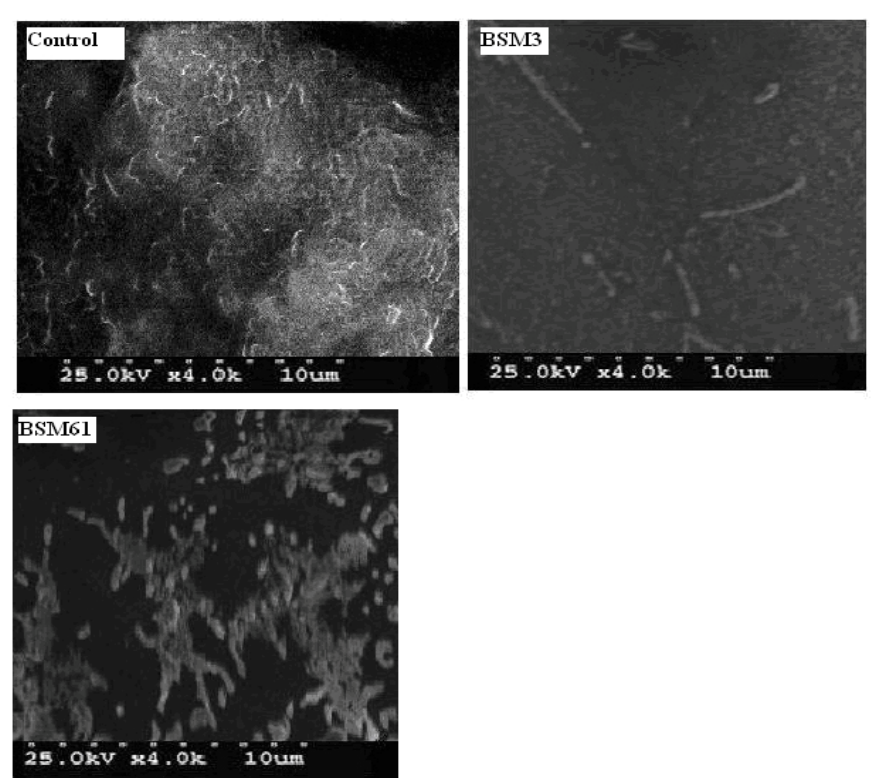

Figure 2: Scanning electron micrographs (4000X) of parent strain and sucrose independent mutants of $S$. mutans; top left panel is control cell (wild type) with strong biofilm, top right panel is biofilm mutant cells (BSM3), bottom left panel is again biofilm defective mutant (BSM61); these mutant showing reduced biofilm. 


\section{Bioinformation Volume 5}

\section{Conclusion:}

We describe the concerted involvement of several proteins in the formation of Streptococcus mutans biofilm. These mutants show low ex-pression of cell surface adhesion proteins. Some of the differentially expressed proteins are basic metabolic proteins, redox proteins and heat shock proteins.

\section{Acknowledgements:}

The authors would like to thank Dr. Shahid Jameel for providing us the 2D-protein gel facility at ICGEB, India. This work was supported from the DST grant $100 / \mathrm{IFD} / 5160 / 2007-2008$ to AUK. BI, SNK and MA acknowledge SRF from CSIR, India.

\section{References:}

[1] A Yoshida \& HK Kuramitsu, Appl Environ Microbiol. (2002) 68: 6283 [PMID: 12450853]

[2] M Shemesh et al. Microbiology (2007) 153: 1307 [PMID: 17965356]

[3] C Rathsam et al. J Proteome Res. (2005) 4: 2161 [PMID: 16335963]

[4] D Ajdic \& VT Pham, J Bacteriol. (2007) 189: 5049 [PMID: 17496079]

[5] J C Waterhouse et al. Oral Microbiol Immunol. (2007) 22: 103 [PMID: 17311633]

[6] H Murchison et al. Infect Immun. (1981) 34: 1044 [PMID: 7333663$]$
[7] H Murchison et al. Infect Immun. (1982) 38: 282 [PMID: 6183211]

[8] KK Jefferson, FEMS Microbiol Lett. (2004) 236: 163 [PMID: 15251193]

[9] N S Jakubovics et al. Mol Microbiol. (2005) 55: 1591 [PMID: 15720563]

[10] B Islam et al. Med Sci Monit. (2007) 13: 196 [PMID: 17968308]

[11] A Yano et al. FEMS Microbiol Lett. (2002) 217: 23 [PMID: 12445641]

[12] S Hamada et al. Infect Immun. (1981) 32: 364 [PMID: 6452415]

[13] R P Shellis et al. Journal of Dentistry (2005) 33: 313 [PMID: 15781139]

[14] N S Jakubovics et al. Infect Immun. (2005) 73: 6629 [PMID: 16177339]

[15] B Islam et al. J Antimicrob Chemother. (2008) 62: 751 [PMID: 18565974]

[16] H Lowry et al. J Biol Chem. (1951) 193: 265 [PMID: 14907713]

[17] PH O' Farrell, J Biol Chem. (1975) 250: 4007 [PMID: 236308]

[18] M Berth, Appl Microbiol Biotechnol. (2007)76: 1223 [PMID: 17713763]

[19] A Carlen et al. J Dent Res. (1998) 77: 81 [PMID: 9437403]

[20] S J Ahn et al. Infect Immun.. (2008) 76: 4259 [PMID: 18625741]

[21] S Shintani et al. Cancer Sci. (2003) 94: 894 [PMID: 14556663]

[22] G Svensäter et al. Microbiology (2000) 146: 107 [PMID: 10658657]

Edited by $\mathbf{P}$ Kangueane Citation: Khan et al. Bioinformation 5(10): 440-445 (2011) License statement: This is an open-access article, which permits unrestricted use, distribution, and reproduction in any medium, for non-commercial purposes, provided the original author and source are credited. 


\section{Supplementary material:}

Table 1: Identification of at least five folds differentially expressed proteins in parent strain (P) and mutants BSM3, BSM5 and BSM61 (mean of the mutants $\mathrm{M}$ )

\begin{tabular}{|c|c|c|c|c|c|}
\hline Spot ID & $\mathrm{pI}$ & MW(Da) & Ratio(P/M) & Gene ID & Protein identified \\
\hline 1 & 5.35 & 80608 & -9.79 & Not identified & \\
\hline 2 & 4.5 & 80291 & -9.54 & Not identified & \\
\hline 3 & 5.19 & 81285 & -8.35 & Not identified & \\
\hline 4 & 5.28 & 81034 & -7.96 & Not identified & \\
\hline 5 & 5.49 & 81430 & -7.96 & SMu0610 & nrdE ribonucleotide reductase, large subunit \\
\hline 6 & 5.72 & 80537 & -7.67 & Not identified & \\
\hline 7 & 4.95 & 80784 & -6.30 & Not identified & \\
\hline 8 & 4.86 & 80537 & -6.22 & Not identified & \\
\hline 9 & 4.14 & 77912 & -5.90 & Not identified & \\
\hline 10 & 5.54 & 10945 & -5.48 & Smu1499 & Rex A transcriptional regulator \\
\hline 11 & 5.30 & 21017 & -5.28 & Smu1446 & conserved hypothetical protein \\
\hline 12 & 4.00 & 77875 & -5.25 & Not identified & \\
\hline 13 & 4.34 & 1784 & -5.04 & Not identified & \\
\hline 14 & 4.70 & 41825 & 5.12 & Smu0240 & agmatine depimerase \\
\hline 15 & 4.77 & 3100 & 5.47 & Not identified & \\
\hline 16 & 4.71 & 14667 & 5.65 & Smu0819 & conserved hypothetical protein \\
\hline 17 & 6.04 & 29697 & 5.67 & Smu1377c & hypothetical protein \\
\hline 18 & 4.32 & 41903 & 5.75 & Smu1538 & Glucose-1-phosphate adenylyl transferase \\
\hline 19 & 6.34 & 41658 & 5.84 & Smu0780 & aroC chorismate synthase \\
\hline 20 & 5.47 & 43565 & 5.91 & Smu0537 & $\operatorname{trpB}$ \\
\hline 21 & 5.29 & 42413 & 6.04 & Smu1505 & D-3 phosphoglycerate dehydrogenase \\
\hline 22 & 5.87 & 15160 & 6.42 & Smu0443 & General stress protein \\
\hline 23 & 6.13 & 43399 & 6.43 & Smu1311 & UDP-N-acetyl glucosamine-2-epimerase \\
\hline 24 & 4.61 & 11087 & 6.50 & Smu1432 & hypothetical protein \\
\hline 25 & 4.85 & 13601 & 6.64 & Smu0743 & large conductance mechanosensitive channel \\
\hline 26 & 4.59 & 65280 & 6.65 & Smu004 & Chaperone protein Dna K \\
\hline 27 & 5.05 & 31065 & 7.19 & Smu0422 & $\mathrm{NAD}(+)$ 11C-component synthetase \\
\hline 28 & 4.69 & 41074 & 7.33 & Smu0004 & uncharacterized GTP-binding protein \\
\hline 29 & 6.04 & 45464 & 7.38 & Smu0226 & nifS \\
\hline 30 & 5.40 & 43994 & 8.30 & Smu1128 & Phosphopentomutase \\
\hline 31 & 5.50 & 39717 & 9.19 & Smu0107 & Alcohol dehydrogenase class III \\
\hline 32 & 4.55 & 59637 & 9.67 & Smu0208 & conserved hypothetical protein \\
\hline 33 & 5.10 & 43900 & 9.74 & Smu0300 & arginosuccinate synthase \\
\hline 34 & 5.61 & 42251 & 9.86 & Not identified & \\
\hline 35 & 5.10 & 29734 & 10.64 & Smu0407 & Gamma glutamyl kinase \\
\hline 36 & 5.92 & 29978 & 11.06 & Smu1183 & glutathione -S- transferase \\
\hline 37 & 6.20 & 44705 & 14.36 & Smu1429 & UDP-N-acetylglucosamine 1-carboxyvinyltransferase \\
\hline 38 & 4.82 & 43674 & 15.35 & Smu1097 & $30 \mathrm{~S}$ ribosomal protein $\mathrm{S} 1$ \\
\hline 39 & 6.41 & 18396 & 17.06 & Smu08852 & 2-amino-4-hydroxy-6-hydroymethyldihydropteridine pyrophosphokinase \\
\hline 40 & 5.21 & 10989 & 18.11 & Smu0357 & conserved hypothetical protein \\
\hline 41 & 6.43 & 45033 & 20.44 & Smu0766 & thiamine biosynthesis \\
\hline 42 & 4.82 & 40111 & 20.87 & Smu0454 & hypothetical protein \\
\hline 43 & 5.49 & 41245 & 25.48 & Not identified & \\
\hline 44 & 6.15 & 40192 & 28.93 & Smu0783 & carbamoyl-phosphate synthase \\
\hline 45 & 5.30 & 41689 & 38.63 & Smu0286 & hippurate hydrolase-peptidase aminocyclase \\
\hline 46 & 4.71 & 23800 & 86.57 & Smu0447 & transaldolase family protein \\
\hline 47 & 5.72 & 43480 & 241.81 & Smu1293 & coproporphyrinogen III oxidase \\
\hline 48 & 5.60 & 41130 & 540.52 & Smu0608 & N-acetylornithine aminotransferase \\
\hline 49 & 4.49 & 30335 & $\alpha$ & Smu0388 & hypothetical protein \\
\hline 50 & 5.12 & 41389 & $\alpha$ & Smu1892 & $\operatorname{rec} \mathrm{A}$ \\
\hline 51 & 6.23 & 44270 & $\alpha$ & Smu0767 & CapA \\
\hline 52 & 5.81 & 42703 & $\alpha$ & Smu0394 & N-acetylglucosamine 6-phosphate deacetylase \\
\hline 53 & 5.61 & 45219 & $\alpha$ & Smu1109 & Dehydrooratase \\
\hline 54 & 5.12 & 41665 & $\alpha$ & Smu1945 & tRNA (5-methylaminmethyl-2-thiouridylate)-methyltransferase \\
\hline 55 & 5.67 & 41373 & $\alpha$ & Smu0118 & aminoacid aminohydrolase-hippurate aminohydrolase \\
\hline 56 & 6.00 & 42325 & $\alpha$ & Smu1398 & glucogen biosynthesis protein \\
\hline 57 & 5.92 & 45574 & $\alpha$ & Smu1386 & UDP-N-acetylglucosamine 1-carboxyvinyltransferase \\
\hline
\end{tabular}




\section{Bioinformation}

Table 2: Initial adherence of the sucrose-independent mutants

\begin{tabular}{ll}
\hline Candidate mutants & Percent initial adherence \\
\hline BSM3 & $25.4 \pm 0.2$ \\
BSM10 & $32.6 \pm 0.1$ \\
BSM5 & $31.2 \pm 0.3$ \\
BSM29 & $29.8 \pm 0.3$ \\
BSM13 & $34.2 \pm 0.3$ \\
BSM23 & $39.6 \pm 0.4$ \\
BSM61 & $46.2 \pm 0.3$ \\
BSM20 & $43.3 \pm 0.2$ \\
BSM9 & $38.1 \pm 0.3$ \\
BSM11 & $41.8 \pm 0.1$ \\
BSM16 & $35.4 \pm 0.1$ \\
BSM33 & $27.8 \pm 0.2$ \\
\hline
\end{tabular}

The OD630nm of the cells was read after release of the crystal violet dye using $7 \%$ acetic acid. The OD630nm of the control regarded as $100 \%$ was subsequently used to calculate the percent initial adherence

Table 3: Percent biofilm formation for the parent strain and the sucrose-independent mutants

\begin{tabular}{lllll}
\hline Candidate mutants & \multicolumn{4}{c}{ Percent biofilm formation } \\
\cline { 2 - 5 } & $6 \mathrm{~h}$ & $12 \mathrm{~h}$ & $20 \mathrm{~h}$ & $24 \mathrm{~h}$ \\
\hline Parent strain & 22.89 & 86.74 & 95.18 & 100 \\
BSM3 & 10.22 & 25.61 & 29.98 & 32.34 \\
BSM5 & 12.26 & 33.15 & 37.23 & 39.17 \\
BSM9 & 15.23 & 38.51 & 41.8 & 44.21 \\
BSM10 & 12.99 & 34.25 & 38.39 & 43.33 \\
BSM13 & 13.65 & 35.55 & 40.26 & 42.98 \\
BSM16 & 14.54 & 37.12 & 40.43 & 42.55 \\
BSM11 & 16.72 & 42.12 & 46.67 & 48.92 \\
BSM20 & 16.95 & 43.73 & 48.72 & 51.34 \\
BSM23 & 15.97 & 40.24 & 44.13 & 46.65 \\
BSM29 & 11.52 & 27.29 & 32.24 & 35.49 \\
BSM33 & 10.98 & 27.65 & 30.88 & 33.16 \\
BSM61 & 17.09 & 45.25 & 49.08 & 53.27 \\
\hline
\end{tabular}

The maximum biofilm formation by the parent control strain at $24 \mathrm{~h}$ was assumed to be 100 to calculate the percent biofilm formation at different phases by the parent as well as mutant strain.

Table 4: Average percent biofilm formation by the parent strain (PS), sucrose-independent adherence defective mutants (SI) and sucrose-dependent adherence defective mutant strains (SD) at varied phases of growth

\begin{tabular}{llll}
\hline Incubation time $(\mathrm{h})$ & \multicolumn{3}{c}{ Percent biofilm formation } \\
\cline { 2 - 4 } & PS & SI* & SD** \\
\hline 6 & 22.89 & 14.01 & 22.54 \\
12 & 86.74 & 35.87 & 52.17 \\
20 & 95.18 & 39.98 & 57.31 \\
24 & 100 & 42.78 & 58.31 \\
\hline
\end{tabular}

*represents the average result of 12 sucrose-independent mutants **represents the average result of 18 sucrose-dependent mutants

Table 5: Thickness of the biofilm viewed by CLSM

\begin{tabular}{ll}
\hline Name of the mutant & Thickness of the biofilm $(\mu \mathrm{m})$ \\
\hline Parent strain & $24.5 \pm 0.4$ \\
BSM3 & $12.9 \pm 0.3$ \\
BSM5 & $14.7 \pm 0.5$ \\
BSM61 & $17.5 \pm 0.6$ \\
\hline
\end{tabular}

